\title{
Osteopathic treatment in a patient with left-ventricular assist device with left brachialgia: a case report
}

This article was published in the following Dove Press journal:

International Medical Case Reports Journal

13 January 2017

Number of times this article has been viewed

\section{Bruno Bordoni ${ }^{1-3}$ \\ Fabiola Marelli ${ }^{2,3}$ \\ Bruno Morabito ${ }^{2-4}$ \\ Beatrice Sacconi ${ }^{5}$}

'Foundation Don Carlo Gnocchi IRCCS, Department of Cardiology, Institute of Hospitalization and Care with Scientific Address, Milan, ${ }^{2}$ CRESO, School of Osteopathic Centre for Research and Studies, Gorla Minore, ${ }^{3}$ CRESO, School of Osteopathic Centre for Research and Studies, Falconara Marittima, ${ }^{4}$ Department of Radiological, Oncological and Anatomopathological Sciences, Sapienza University of Rome, ${ }^{5}$ Center for Life Nano Science, CLNS@Sapienza, Istituto Italiano di Tecnologia, Rome, Italy
Correspondence: Bruno Bordoni Foundation Don Carlo Gnocchi IRCCS, Department of Cardiology, Institute of Hospitalization and Care with Scientific Address, Santa Maria Nascente, Via Capecelatro 66, Milan 20100, Italy $\mathrm{Tel}+393496300617$

Email bordonibruno@hotmail.com

\begin{abstract}
This study deals with an osteopathic approach used for a patient with left-ventricular assist device (L-VAD) affected by left brachialgia. Clinical examination revealed the presence of thoracic outlet syndrome and pectoralis minor syndrome, with compression of the left proximal ulnar nerve, related to the surgical sternotomy performed. The osteopathic techniques used can be classified as indirect and direct, addressed to the pectoralis minor and the first left rib, respectively. To our knowledge, this is the first text in literature with an osteopathic treatment in a patient with L-VAD.
\end{abstract}

Keywords: osteopathic, L-VAD, thoracic outlet syndrome, TOS, myofascial, fascia

\section{Introduction}

Chronic heart failure (CHF) is a condition characterized by the inability of the heart to ensure proper supply of oxygen to the body tissues. ${ }^{1}$ One of the most innovative and latest therapies is the treatment with stem cells to restore cardiac tissue, but lack sufficient data to be able to follow this line of care. ${ }^{2}$ When heart failure is severe, the patient may be subjected to transplant or implant of artificial heart. There is not always a number of organs that corresponded to transplant demand, and one of the most promising solutions is the implant of mechanical ventricular support system. ${ }^{3}$ The most commonly surgically implanted device is left-ventricular assist device (L-VAD), a mechanical pump that supports the cardiac pump function, intervening in support of left ventricle in severe dysfunction, and which, in the newer models, provides a continual flow. ${ }^{3}$ Connected to a pump, there is a cannula for the outflow that exits from the aorta and one for the inflow, which enters the apex of the left ventricle. A package of external batteries for the operation of the pump is put into connection by means of an electric wire, called the drive line, which exits at the level of the subcutaneous tissue of the chest or peritoneum. ${ }^{4}$ Because the system is carried out with the ultimate aim of having a transplant, it is referred to as "bridge therapy", while if the use of L-VAD becomes a definitive therapeutic solution, it is described as a "destination therapy". ${ }^{4,5}$ From 2006 to 2013, more than 2500 devices have been implanted around the world, with a survival rate of $81 \%$ after 1 -year postimplantation; the survival of patients with this method improves the quality of life and gives access to rehabilitation programs. ${ }^{6}$

Patients undergoing a sternotomy may present transient or permanent injury to the brachial plexus and trauma to the first rib, probably because of the position 


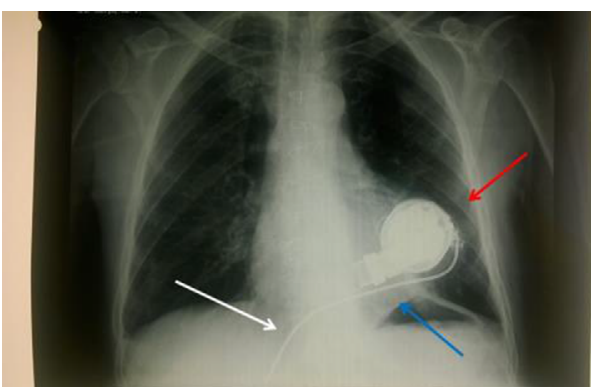

Figure I The X-ray shows the internal turbine with a first tube inside the left ventricle, the drive connecting it to the outside, and the left tube that will go into the aorta.

Notes: There are no signs of fracture of the first rib. The $X$-ray was made on the second day of hospitalization. Red arrow denotes mechanical pump, blue arrow denotes left ventricle, and white arrow denotes drive line.

maintained during surgery (with the arm being placed in abduction over the head), and due to the sternal retractor (Figure 1). ${ }^{7,8}$

\section{Case presentation}

A 51-year-old patient with L-VAD was admitted to our clinic 4 months after surgery, due to repeated episodes of infection (Pseudomonas aeruginosa and Citrobacter freundii). The patient had undergone an anterior infarction with cardiac arrest, with a reduction in the ejection frequency $<20 \%$. A HeartMate III L-VAD type was implanted. As risk factors, the patient had a family history of cardiovascular disease and smoking; he suffered from hiatal hernia and underwent a previous lumbar discectomy. Until his acute cardiac event, the patient had a normal active daily routine, working and having a regular physical activity.

The X-ray radiogram performed in our clinic showed correct positioning of the device and no pleural effusion; saturation was $\sim 99 \%$, and blood pressure values were within normal limits. Its medical therapy included cardioaspirin, amiodarone, digoxin, furosemide, omeprazole, potassium, ramipril, simvastatin, and sintrom.

After surgery, the patient complained with a persistent left brachialgia, presenting throughout the whole day and night. A triad of symptoms typical of a neurogenic compression was present: pain, weakness, and paresthesia. ${ }^{9}$ The anamnesis of the patient and the craniosacral palpation guided our attention to the thoracic outlet. The pain was perceived in the region of the left trapezius, of the cervical spine, and supraclavicular and axillar area; paresthesias and weakness involved the last two fingers of the left hand. Signs of ulnar sufferance were observed at physical examination, in the presence of thoracic outlet syndrome (TOS) and pectoralis minor syndrome (PMS), with the typical clinical signs described in literature. ${ }^{9}$ Tinel's sign was positive at the level of the left medial epicondyle of the left medial and of the ulnar tunnel (Guyon's canal, between the pisiform bone and the hook of uncinate bone); we actively and passively examined the articulation of the elbow and forearm, without finding movement restrictions. Overtone of the left trapezius muscle and the anterior scalene was observed and overtone of the left pectoralis minor and subclavian muscles was observed; to check the tone of the muscles, we used the modified Ashworth scale (score of 2). This scale allows to evaluate the tone of muscles, using passive and active movements of the muscle groups, giving a value to the tension recorded by the operator. ${ }^{10}$

The provocative maneuvers such as upper limb tension test (ULTT) of Elvey and elevated arm stress test (EAST) of Roos resulted positive. The first rib left was in inspiratory attitude, and a limited range of motion (ROM) was observed in the cervico-dorsal junction and in the artrocinematic of the left shoulder. To evaluate the first rib bilaterally, using your index fingers, you must palpate the root of the neck in front of the trapezius muscle, where there is a bone formation (the medial aspect of the first rib) that changes its position according to whether the patient is inhaling or exhaling: it rises during inhalation and lowers during exhalation. ${ }^{11}$ If the first rib is not lowered during expiration, it is in inspiratory attitude. The patient signed an informed consent with permission to use the photographs and data; all patients of our clinic are evaluated and are subjected to standard treatment, according to the Declaration of Helsinki - Ethical Principles for Medical Research Involving Human Subjects.

\section{Osteopathic treatment}

After clinical evaluation, it was decided to approach the patient with two osteopathic techniques: an indirect technique for the pectoralis minor and a direct technique for the first rib.

The indirect osteopathic approach or myofascial release aims to release fascial restrictions, to mobilize tight ligaments, to drain congested lymphatics, and to alter the mechanical properties of fascia, such as density, stiffness, and viscosity, so that the fascia can more readily adapt to physical stresses. ${ }^{12}$ In fact, some osteopathic physicians and manual therapists report local tissue release after the application of a slow manual force to tight fascial areas; these reports have been explained as a breaking of fascial cross-links, a transition from gel-to-sol state in the extracellular matrix and other passive viscoelastic changes of fasciae. ${ }^{12}$ The fascial osteopathic technique is the application of a low-load, long-duration stretch into the myofascial complex, with the aim to restore the optimal length of this complex. The practitioner palpates 
the fascial restriction, and the pressure is applied directly to the skin, into the direction of restriction, until resistance (the tissue barrier) is felt. Once found, the collagenous barrier is engaged for 90-120 seconds, without sliding over the skin or forcing the tissue, until the fascia complex starts to yield and a sensation of softening is achieved. ${ }^{12}$ In vitro studies demonstrated how the osteopathic techniques can influence the metabolic behavior of fibroblasts, in terms of proliferation and inflammatory response. ${ }^{12}$ It is proved that, by applying this method, fibroblasts are able to change their orientation and probably their mechanical behavior. ${ }^{12}$ An improved sliding of the various fascial layers would allow to reset the afferent of the free nerve endings, resulting in physiologic response of the efferent ones. ${ }^{12}$

Direct techniques are defined as high-velocity low amplitude (HVLA), which uses a short strength and works in short ranges inside the anatomic articular movement, involving the dysfunctional barrier to promote the release of disfunction. ${ }^{13}$ They are mainly used for the articular areas to improve the amplitude of the ROM and to reduce pain. ${ }^{13}$ The technique is often associated with an audible articular "pop", probably due to a cavitation effect, involving the synovial fluid and gas, and a rapid increase in the articular volumes induced by the technique creates a reduction in the carbon dioxide content in the synovial tissue, resulting in gas bubbles within the joint. ${ }^{13}$ The synovial fluid flow in the low-pressure regions of the cavity collapses the gas bubbles, producing the typical "pop" sound. ${ }^{13}$

A recent review of the literature on this osteopathic approach has demonstrated the ability to inhibit the pain, probably involving descendant's inhibitory mechanisms, more in detail activating the periaqueductal gray area of the midbrain with efferent inhibitory until spinal cord and inhibiting the nociceptive afferents. ${ }^{14}$

The first manual therapeutic step was performed to release the tension of the left pectoralis minor, through an osteopathic approach performed with indirect techniques of myofascial release with the patient being supine. The thumb has to be put under the axilla, with cranial direction toward the coracoid insertion of the muscle, with the force vector being in the oblique axis; once the hypertonic area is identified, under the pectoralis major, this position needs to be maintained until myofascial release occurs (Figure 2).

The next step included an HVLA to release the first left rib in an inspiratory attitude (elevation), with the patient being supine. The left hand of the operator fixed the first rib at the level of the supraclavicular fossa of the trapezius muscle, whereas the other hand grabs the cervico-occipital junction, creating a passive tilt to the left side and a rotation

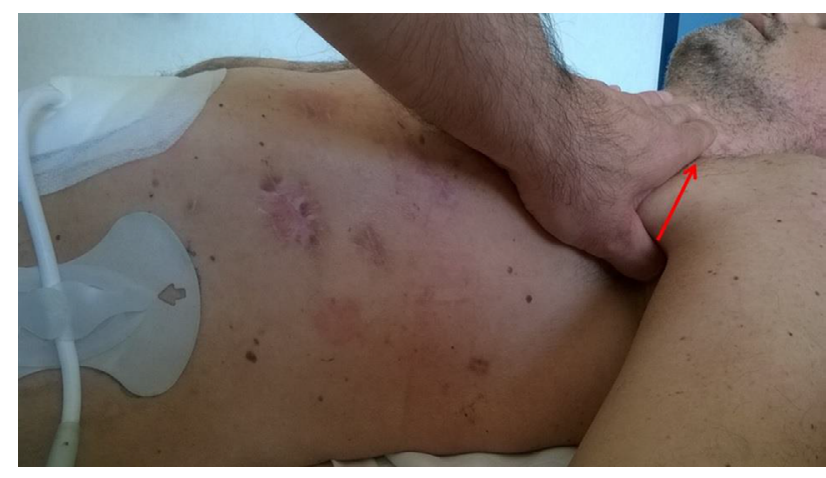

Figure 2 The thumb has to be put under the axilla, with cranial direction toward the coracoid insertion of the muscle, with the force vector being in the oblique axis; once identified the hypertonic area, under the pectoralis major, this position needs to be maintained until myofascial release occurs.

Note: The red arrow indicates the direction of the thumb, to the coracoid insertion.

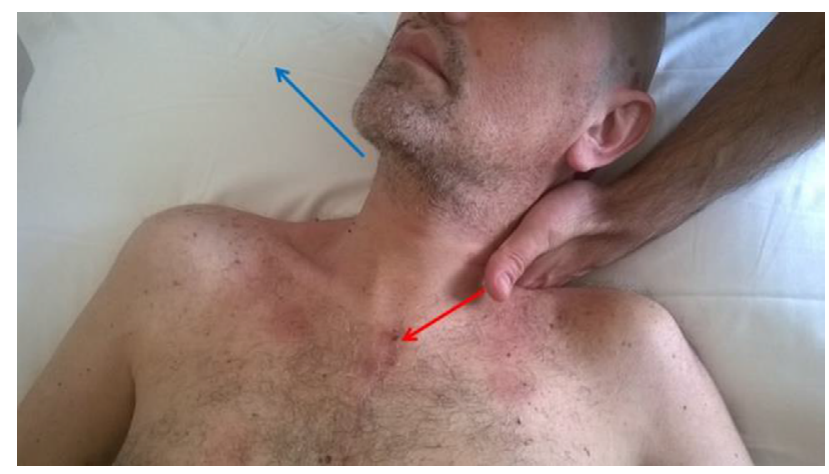

Figure 3 The left hand of the operator fixed the first rib at the level of the supraclavicular fossa of the trapezius muscle, while the other hand grabs the cervicooccipital junction, creating a passive tilt to the left side and a rotation to the right side; this movement needs to stretch the DI costovertebral joint, limiting parasitic movements and avoiding the involvement of other joints.

Note: To increase the tension, the patient was asked to inhale; the first rib was pushed caudally (red arrow), at the same time giving a rotational impulse of a few degrees (blue arrow), until obtaining the costal "pop".

to the right side; this movement needs to stretch the D1 costovertebral joint, limiting parasitic movements and avoiding the involvement of other joints. To increase the tension, the patient was asked to inhale; the first rib was pushed caudally, at the same time giving a rotational impulse of a few degrees, until obtaining of the costal "pop" (Figure 3).

The treatment lasted a few minutes, without any adverse events or worsening of the symptoms. At the clinical evaluation performed the next day, pain and muscle hypertone (Ashworth score of 1) were no longer observed, as well as the paresthesias; a slight weakness of the last two fingers persisted (and then disappeared after a week from treatment). The Tinel test, the ULTT, and the EAST resulted negative; there was no limitation of ROM of the cervico-dorsal junction and of the left shoulder; the first rib showed regular movements. The repetition of the evaluations performed by cardiologist, such as echocardiography, showed no clinical and objective signs of a worsening. 


\section{Discussion}

TOS and PMS are commonly observed in the same patient, in the so-called double crush syndrome. ${ }^{9}$ The vascular-nervous brachial plexus passes between the anterior scalene and the middle one, between the first rib and the clavicle, and under the pectoralis minor; a compression at these levels will create neurological and/or vascular symptoms. ${ }^{9}$ One of the causes of compression is related to the positioning of the shoulder in complete abduction and anterior rotation during surgical sternotomy; this event will cause a persistent elevation of the first rib, compression of the costo-clavicular nervous junction, and a reduction in the posterior space of the pectoralis minor with hypertone, resulting in compression of the brachial plexus. ${ }^{9,15}$ The case described in this study reports all signs of neurogenic pain of the brachial plexus and, more in detail, of the ulnar nerve for the involvement of the last two fingers of the left hand..$^{7-9}$

The treatment of the pectoralis minor in case TOS/PMS is already used and known to be effective, with immediate symptoms resolution. ${ }^{15,16}$ However, the use of HVLA on the first rib is less common in these syndromes. ${ }^{13}$

The reasons for the disappearance of brachialgia are related to osteopathic treatment. The manual myofascial release treatment is effective in reducing the presence of soft tissue pain, regardless of the body region, although the specific reasons are not fully known. ${ }^{17}$ The parasympathetic nervous system is probably directly involved, decreasing the tone of the tissue and reducing the level of proinflammatory cytokines. ${ }^{18}$ The reduction of the pectoralis minor tension resulted in a larger space for a proper passage of the vascular-nervous brachial plexus.

When a joint is blocked, local pain is induced, also involving the adjacent tissues. ${ }^{13}$ The direct techniques are able to improve the local perception of pain. ${ }^{19}$ The causes are related to the ability of the nervous system to induce hypoalgesia, through mechanical peripheral stimulation. ${ }^{14}$ In addition, the release of the first rib led to an easier passage of the brachial plexus in the costo-clavicular space.

\section{Conclusion}

This report describes the resolution through osteopathic approach of brachialgia caused by TOS/PMS in a patient with L-VAD. The disappearance of symptoms occurred as a result of manual techniques addressed to the pectoralis minor and to the first rib, with myofascial release techniques and joint release. To our knowledge, this is the first study in literature dealing with the osteopathic treatment of a patient with L-VAD.

\section{Disclosure}

The authors report no conflicts of interest in this work.

\section{References}

1. Ramani GV, Uber PA, Mehra MR. Chronic heart failure: contemporary diagnosis and management. Mayo Clin Proc. 2010;85(2):180-195.

2. Le T, Chong J. Cardiac progenitor cells for heart repair. Cell Death Discov. 2016;2:16052.

3. Mallidi HR, Anand J, Cohn WE. State of the art of mechanical circulatory support. Tex Heart Inst J. 2014;41(2):115-120.

4. Brouwers C, Denollet J, de Jonge N, Caliskan K, Kealy J, Pedersen SS. Patient-reported outcomes in left ventricular assist device therapy: a systematic review and recommendations for clinical research and practice. Circ Heart Fail. 2011;4(6):714-723.

5. Yamakawa M, Kyo S, Yamakawa S, Ono M, Kinugawa K, Nishimura T. Destination therapy: the new gold standard treatment for heart failure patients with left ventricular assist devices. Gen Thorac Cardiovasc Surg. 2013;61(3):111-117.

6. Holman WL, Acharya D, Siric F, Loyaga-Rendon RY. Assessment and management of right ventricular failure in left ventricular assist device patients. Circ J. 2015;79(3):478-486.

7. Healey S, O'Neill B, Bilal H, Waterworth P. Does retraction of the sternum during median sternotomy result in brachial plexus injuries? Interact Cardiovasc Thorac Surg. 2013;17(1):151-157.

8. Gupta A, Jamshidi M, Rubin JR. Traumatic first rib fracture: is angiography necessary? A review of 730 cases. Cardiovasc Surg. 1997;5(1):48-53.

9. Sanders RJ, Annest SJ. Thoracic outlet and pectoralis minor syndromes. Semin Vasc Surg. 2014;27(2):86-117.

10. Bakheit AM, Maynard VA, Curnow J, Hudson N, Kodapala S. The relation between Ashworth scale scores and the excitability of the alpha motor neurones in patients with post-stroke muscle spasticity. J Neurol Neurosurg Psychiatry. 2003;74(5):646-648.

11. Korbon GA, Carron H, Lander CJ. First rib palpation: a safer, easier technique for supraclavicular brachial plexus block. Anesth Analg. 1989;68(5):682-685.

12. Bordoni B, Zanier E. Understanding fibroblasts in order to comprehend the osteopathic treatment of the fascia. Evid Based Complement Alternat Med. 2015;2015:860934.

13. Chila AG, editor. Foundations of Osteopathic Medicine. 3rd ed. Philadelphia, PA: Wolters Kluwer Health/Lippincott Williams \& Wilkins; 2011.

14. Savva C, Giakas G, Efstathiou M. The role of the descending inhibitory pain mechanism in musculoskeletal pain following high-velocity, low amplitude thrust manipulation: a review of the literature. $J$ Back Musculoskelet Rehabil. 2014;27(4):377-382.

15. Sucher BM. Ultrasonography-guided osteopathic manipulative treatment for a patient with thoracic outlet syndrome. JAm Osteopath Assoc. 2011;111(9):543-547.

16. Sucher BM. Thoracic outlet syndrome - a myofascial variant: Part 2. Treatment. J Am Osteopath Assoc. 1990;90(9):810-812, 817-823.

17. Ajimsha MS, Al-Mudahka NR, Al-Madzhar JA. Effectiveness of myofascial release: systematic review of randomized controlled trials. J Bodyw Mov Ther. 2015;19(1):102-112.

18. Bordoni B, Marelli F. The fascial system and exercise intolerance in patients with chronic heart failure: hypothesis of osteopathic treatment. J Multidiscip Healthc. 2015;8:489-494.

19. von Heymann WJ, Schloemer P, Timm J, Muehlbauer B. Spinal highvelocity low amplitude manipulation in acute nonspecific low back pain: a double-blinded randomized controlled trial in comparison with diclofenac and placebo. Spine (Phila Pa 1976). 2013;38(7):540-548. 
International Medical Case Reports Journal

The International Medical Case Reports Journal is an international, peer-reviewed open-access journal publishing original case reports from all medical specialties. Previously unpublished medical posters are also accepted relating to any area of clinical or preclinical science. Submissions should not normally exceed 2,000 words or
4 published pages including figures, diagrams and references. The manuscript management system is completely online and includes a very quick and fair peer-review system, which is all easy to use. Visit $\mathrm{http}: / /$ www.dovepress.com/testimonials.php to read real quotes from published authors.

Submit your manuscript here: https://www.dovepress.com/international-medical-case-reports-journal-journal 